\title{
Retrobulbar versus Subconjunctival Anaesthesia for Cataract Surgery - Experience in Nigeria
}

\author{
Joseph Momodu Waziri-Erameh MB, BS FMC(OPH,) FWACS, FICS; ${ }^{1} \mathrm{R}$ Valentina Okeigbemen \\ MBBS, FMCOph; ${ }^{1}$ Ireju Onyinye Chukwuka MBBS, FMC(OPH); ${ }^{2}$ Sydney Ejimadu MBBS, \\ AFMC(OPH) $)^{2}$
}

${ }^{1}$ Department of Ophthalmology, University of Benin Teaching Hospital, Benin City, Nigeria, ${ }^{2}$ Department of Ophthalmology, University of Port Harcourt Teaching Hospital, Port Harcourt, Nigeria

\section{Abstract}

Aim: To test the efficacy of subconjunctival anaesthesia (SCA) for cataract surgery against the established retrobulbar anaesthesia (RBA).

Methods: This was a prospective study of 73 adults (44 males, 29 females) selected for cataract surgery and intraocular lens (IOL) implants under local anaesthesia. Their ages ranged from 24 years to 73 years. Thirty-seven (37) eyes had subconjunctival anaesthesia while 37 had retrobulbar anaesthesia for the cataract surgery and intraocular lens implant. The anaesthetic used was 2\% lignocaine. An agreed marking scheme was formulated to assess the benefits of the anaesthesia and akinesia produced by the both methods. The anaesthesia (both methods) for all the patients was administered by one ophthalmologist and the assessment was done by the ophthalmologist and an assisting ophthalmologist. The results were collated and analysed according to the type of anaesthesia; any complications related to anaesthesia were noted.

Results: Both SCA and RBA were satisfactory for cataract surgery. The RBA was observed to give better akinesia and less photophobia than the SCA during cataract surgery. There were no significant anaesthesia-related complications in either SCA or RBA.

Conclusion: Subconjunctival anaesthesia is not as good as retrobulbar anaesthesia for cataract surgery in Nigeria because of observed less akinesia and more photophobia. There may be a need to give verbal instructions to the patients which they may not understand.

Key words: subconjunctival, retrobulbar, akinesia, anaesthesia, photophobia

\section{INTRODUCTION}

Local anaesthesia has largely replaced general anaesthesia in cataract surgery in adult patients, where the surgical eye is numbed and immobilized while the patient is awake. The advantages of local anaesthesia include not starving the patient, and it eliminates the need for routine investigations such as chest X-ray and electro-cardiogram (ECG). ${ }^{1}$ Elderly patients with senile cataract are also spared the additional risk of general anaesthesia associated with advancing age. Local anaesthesia is less expensive, which is an important consideration in developing countries like Nigeria. The requirements for intraocular surgery using local anaesthesia as established in the 1950s and 1960s are threefold: globe and conjunctival anaesthesia; globe, lid and periorbital akinesia; and intraocular hypotonia. ${ }^{2}$ Techniques of local anaesthesia for cataract surgery include retrobulbar, peribulbar, subtenon, and subconjunctival injections, and topical / intracameral application of local anaesthetic. Retrobulbar and peribulbar are the most popular techniques at present.

Retrobulbar anaesthesia (RBA) - also known as intraconal - is one of the preferred and most effective techniques for local anaesthesia in ocular surgery. ${ }^{3}$ It was first described by Knapp of New York in 1884 for enucleation of an eyeball. ${ }^{4}$ It is easy to learn and easy to perform. RBA anaesthetizes the nerves within the cone of muscles in the orbit causing anaesthesia of the peri-limbal conjunctiva, the cornea and the recti-muscles supplied by the $3^{\text {rd }}$ cranial nerve. Despite its effectiveness, it has numerous complications, many of which are serious and some life-threatening. These complications include retrobulbar haemorrhage, globe perforation/penetration, intra-vascular injection leading to central nervous system and cardiac complications, injury to the optic nerve and retinal artery occlusion. ${ }^{5}$

Retrobulbar haemorrhage is the most important and the most common of the serious complications of RBA, ${ }^{6}$ and has

${ }^{*}$ Correspondence: Dr. Joseph Momodu Waziri-Erameh, Department of Ophthalmology, University of Benin Teaching Hospital, Benin City, 
a reported rate of $0.44 \%$ to $1.7 \% .^{7}$ In many cases, retrobulbar haemorrhage leads to the cancellation of surgery, ${ }^{8}$ but successful surgery has been reported, even after retrobulbar haemorrhages. ${ }^{9}, 10$ Bending the needle about 45 degrees midway reduces some of the serious complications, ${ }^{11}$ as the tip of the needle will be far from the orbital apex and floor of the orbit.

Attempts at reducing (or avoiding) the complications of RBA led to the development of peribulbar, subtenon, and subconjunctival injections and other techniques of local anaesthesia for cataract surgery.

Peribulbar anaesthesia became popular because it was said to be safer than RBA, ${ }^{12}$ but it still had many of the serious side effects of RBA, such as globe perforation and penetration, nerve injury and retrobulbar haemorrhage. ${ }^{13}$ It is not as easy to learn and perform as RBA and it is also less effective as RBA. $^{14}$

Subtenon anaesthesia was first used over 100 years ago to carry out an enucleation. ${ }^{15}$ It is reported to have wide applications in ophthalmic surgery including cataract; its anaesthestic attributes are good for the iris, anterior segment and conjunctiva. ${ }^{16}$ The complications associated with subtenon anaesthesia are fairly minor, ${ }^{17}$ but it has the disadvantage of poor akinesia ${ }^{18}$ and requires a special needle. ${ }^{19}$

Subconjunctival anaesthesia (SCA) was reported in 1990 to be effective for cataract surgery. ${ }^{20,21}$ It is very simple and provides fast-acting anaesthesia of the anterior segment and conjunctiva. No special needle is required and it does not cause intra-orbital or intraocular pressure to rise. ${ }^{22}$ The few reported complications are poor akinesia and occasional chemoses. $^{23}$

Mindful of the complications associated with RBA; and the equally serious complications, technical difficulties and less effective attributes of peribulbar anaesthesia; ${ }^{13}$ the technical difficulty / need for special needles for subtenon; and encouraged by the reports on subconjunctival anaesthesia, ${ }^{16,17}$ we decided to carry out a prospective study on the efficacy of subconjunctival anaesthesia (SCA), which is not widely used for cataract surgery in Nigeria against the popular retrobulbar anaesthesia (RBA).

\section{PATIENTS AND METHODS}

This study was designed to compare the efficacy of retrobulbar anaesthesia (RBA) with subconjuctival anaesthesia (SCA) and to study and compare observed anaesthesia-related complications in adult Nigerians who underwent cataract surgery. The design was a prospective tri-centre study carried out at the DDS eye surgeries in Benin, Warri and Port Harcourt (South-South Nigeria). It was a prospective study involving 73 adults selected for cataract surgery in the three centres. The selection criteria were adults 18 years and over who needed to have cataract and intra ocular lens implant surgery under local anaesthesia. Male and female patients were included in this study. The selected patients were subdivided into two groups - RBA and SCA. The age and sex of the patients in the RBA group were matched as much as possible with those in the SCA group. Patients with only one eye, patients with difficulty in lying flat, or patients with major systemic illnesses were excluded. The anaesthetic agent used was $2 \%$ lignocaine diluted with adrenaline at a rate of 1 : 200,000 . The patient's name, age, sex, type of surgery and type of anaesthesia were recorded.

The technique for retrobulbar anaesthesia involved the patients lying face up with the eyes directed at primary gaze position. ${ }^{24}$ The needle for the injection was bent midway about 45 degrees bevel facing up. The bent needle was inserted tangentially through the skin at the infero- temporal side of the lower orbital margin with the bevel facing the globe. No further manipulation of the needle was necessary. The needle follows the path of the bend such that the tip moves away from the periosteum of the orbit the deeper it goes into the orbit and also directs the needle tip away from the apex of the orbit with large veins and optic nerve. Then $3 \mathrm{ml}$ of $2 \%$ lignocaine was injected slowly into the cone formed by the recti-muscles. The eye was then gently and firmly massaged over an eye pad for about one minute.

The subconjunctival anaesthesia (modified version) involved about $1 \mathrm{ml}$ each of $2 \%$ lignocaine injected sub conjunctival and inter recti $(5-7 \mathrm{~mm}$ from the limbus) in the four quadrants with a 27 -gauge needle. No massaging was required.

In both groups, the orbicularis was blocked separately using the Van Lint method near the lateral canthus. The effectiveness of each group was assessed objectively without the patients' input. During the surgery, the grades of anaesthesia and akinesia of the globe were: total, adequate, inadequate, and failed. Total and adequate were classified as satisfactory, while inadequate and failed were classified as unsatisfactory. We also looked for signs of photophobia (increased effort to blink or move globe) after delivering the lens nucleus. Complications observed in any of the groups of anaesthesia were also noted and recorded.

All the anaesthesia was administered by one ophthalmologist, while the assessment of akinesia, anaesthesia and photophobia were independently noted and recorded by the ophthalmologist and the assistant (an ophthalmologist) after a marking scheme had been formulated.

\section{OBJECTIVE MARKING SCHEME}

\section{Akinesia}

Total - Insignificant or no globe movement experienced during surgery 

Adequate - Some globe movements, but no additional anaesthetic injection given for the surgery.
Inadequate - Significant movements; additional anaesthetic injection given for the surgery
Failed
- Surgery not possible with method of anaesthesia alone.

\section{Anaesthesia}

Total

Adequate

Inadequate

Failed

Photophobia

- No obvious response to pain

- Some response to pain such as when handling the conjunctiva but no further anaesthetic required

- Reacts significantly to pain such as when handling cornea or conjunctiva and further injection of anaesthetic required for the surgery

- Surgery not possible with method of anaesthesia alone

- Attempts to shut eyelids against the retractors and or attempt to move eyes because of operating light.

\section{RESULT}

Seventy-three patients (73) aged between 24 and 89 years were involved in this study. The peak age group was 60-69 years accounting for $32 \%$ of all the patients. Males were 44 and females 29 (ratio 1.6:1). Thirty-seven (37) patients were in the RBA group while 36 patients were in the SCA group. The patients were distributed as equally as possible between the two groups according to age and gender.

Tables 1 and 2 show the observed efficacy (akinesia and anaesthesia) and photophobia in the RBA and SCA groups. In the RBA group, 26 patients had total akinesia, 10 patients had adequate akinesia and one patient had inadequate akinesia. As for anaesthesia, 16 patients had total anaesthesia, 20 patients had adequate anaesthesia and one patient inadequate anaesthesia. Only one patient in the RBA group had photophobia.

In the SCA group, no patient had total akinesia; 28 patients had adequate akinesia, 7 patients inadequate akinesia and one patient had failed akinesia No patient had total anaesthesia; 35 patients had adequate anaesthesia, and one patient had inadequate anaesthesia. All the 36 patients experienced some degree of photophobia.

The observed complications were: lid haemorrhage in 6 patients in the RBA group; an increase in intraocular pressure - IOP - (observed during surgery) in 13 patients in the SCA group, and conjunctival haemorrhage in 5 patients.
Table 1. Akinesia in RBA and SCA

\begin{tabular}{lcccc}
\hline Akinesia & RBA $(37)$ & $(\%)$ & SCA $(36)$ & $(\%)$ \\
\hline Total & 26 & 70.3 & 0 & - \\
Adequate & 10 & 27.0 & 28 & 77.8 \\
Inadequate & 1 & 2.7 & 7 & 19.4 \\
Failed & - & - & 1 & 2.8 \\
\hline
\end{tabular}

Satisfactory anaesthesia $=$ sum of total + adequate

Satisfactory anaesthesia : RBA vs SCA, p $=0.154$

Table 2. Anaesthesia in RBA and SCA

\begin{tabular}{lcccc}
\hline Anaesthesia & RBA (37) & $\%$ & SCA (36) & $\%$ \\
\hline Total & 16 & 43.2 & - & - \\
Adequate & 20 & 54.1 & 35 & 97.2 \\
Inadequate & 1 & 2.7 & 1 & 2.8 \\
Failed & - & - & - & - \\
\hline Photophobia & 1 & 2.7 & 36 & 100.0 \\
\hline
\end{tabular}

\section{Discussion}

Local anaesthesia is preferred whenever possible because it is safer, cheaper and quicker, has fewer post-operative complications and can be completely controlled by the surgeon. We observed the efficacy of SCA as an alternative to RBA, which has known serious complications. ${ }^{5,18}$ SCA is effective and is not associated with serious complications and other difficulties, ${ }^{21}$ unlike other alteratives such as peribulbar and subtenon anaesthesia. ${ }^{13,18}$ We however designed this study to establish the response of our largely illiterate/semi-illiterate cataract patients whose co-operation cannot be accurately relied upon. The ophthalmologist administers the local anaesthesia for ocular surgery in Nigeria thus freeing the limited numbers of anaesthetists for surgeons who cannot operate using local anaesthesia. When comparing our results to the literature, our findings are similar. In retrobulbar anaesthesia, the retrobulbar haemorrhage rate ranges for $0.44 \%-1.7 \%$ but very insignificantly low with the bent-needle technique. We had no case of retrobulbar haemorrhage using the bent-needle technique. The bent needle keeps the tip of the needle away from the periosteum of the floor of the orbit. Retrobulbar haemorrhages result when the tip of the needle penetrates the periosteum. With the bent needle, the tip of the needle, in its final position, is far from the apex of the orbit that has large vessels. Lying face up and looking in the primary gaze position, ${ }^{24}$ and the needle inserted tangentially, has fewer complications than the position in which the patient faces up, and looking in (nasal) and the needle directed up and in. ${ }^{25}$ The significant complication encountered in the RBA group was lid haemorrhages $(16.2 \%)$, which were mild ecchymosis. This could be because RBA is like a blind 
procedure in Africans and dark skinned people as the superficial vessels of the lid, which are visible in light- skinned persons are not visible in negroids.

A total of 36 patients each $(97.3 \%$ ) had satisfactory (total and adequate) akinesia and adequate anaesthesia in the RBA group; the SCA group had 28 patients $(77.8 \%)$ with satisfactory akinesia and 35 patients $(97.2 \%)$ with satisfactory anaesthesia. Eight patients $(22.2 \%)$ in the SCA group had unsatisfactory (inadequate and failed) akinesia but the surgery was successfully completed in 7 of them with an additional injection of lignocaine. The RBA group produced more satisfactory akinesia (97.3\%), than the SCA group ( $77.8 \%$ ), which is explained by extra conal local anaesthesia being less effective than intraconal (RBA) ${ }^{4}$ in producing akinesia. When this observation was subjected to statistical analysis, $\mathrm{p}>0.05$, which may be due to the small sample size. The ability to adjust the amount of anaesthestic until adequate motor block is achieved is an advantage in SCA. We used $2 \%$ lignocaine with no bupivacaine added, as $2 \%$ lignocaine, apart for being cheaper for developing countries, has always been adequate for the duration of our cataract surgery which is routinely performed as a sutureless / minimal suture extracapsular cataract extraction technique that has cut surgery time significantly.

Thirty-five eyes $(97.2 \%)$ had satisfactory anaesthesia in the SCA group. This is in line with findings by others that it rapidly produces effective anaesthesia. ${ }^{20,21,22}$ All the patients in the SCA group had some degree of photophobia, as it does not block the optic nerve. ${ }^{17}$ The observed non-serious complications encountered in SCA were conjunctival haemorrhage $13.9 \%$; while $36.1 \%$ had a mild increase in intraocular pressure (IOP) observed by attempts to shut the eyelids due to photophobia during surgery. Only two patients required additional lid injections to further block the orbicularis oculi muscle.

In conclusion, both RBA and SCA are effective (satisfactory) anaesthesia methods for cataract surgery in Nigerian adults. In this study, SCA satisfactorily provided akinesia to $77.8 \%$ of globes, but was less effective than RBA, which provided akinesia to $97.3 \%$ of globes. Although SCA has less serious side effects, it is not recommended in this study as the local anaesthesia of choice for Nigerians because of greater ocular motility (less akinesia) and the high incidence of photophobia, with the associated but mild increase in IOP. This could cause serious intraoperative problems, especially in patients who may not fully comprehend the verbal instructions given to them during the surgery. For now, SCA should be used as a reliable alternative for patients who refuse RBA or have high intraocular pressure, in order to avoid the risk of possible retrobulbar haemorrhage, which would further increase the IOP, or cause the surgery to fail due to associated complications.

\section{REFERENCES}

1. Rubin AP. Anaesthesia for cataract surgery-time to change? Anaesthesia 1990; 45: 717-718 .

2. Afkinson WS. Local anaesthesia in ophthalmology. Am J Ophthalmol 1948; 31: 1607-1618.

3. Hamilton RC. Techniques of orbital regional anaesthesia . $\mathrm{Br} \mathrm{J}$ Anaesth 1995; 75: 88-92

4. Knapp H. On cocaine and its use in ophthalmic and general surgery. Arch Ophthalmol 1884; 13: 402-408.

5. Report of Joint Working Party on Anaesthesia in Ophthalmic Surgery. Royal College of Anaesthetist-College of Ophthalmologist, March 1993.

6. Nicoll JM, Achaya PA, Ahlen K, et al. Central nervous system complications after 600 retrobulbar blocks. Anesth Analg 1987; 66: 1298-1302.

7. Morgan CM, Schatz H, Vine AK, Cantrill HL, Davidorf FH, Gitter KA, Rudich R. Ocular complications associated with retrobulbar injections. Ophthalmology 1988; 95: 660-665.

8. Edge KR, Nicoll JM. Retrobulbar haemorrhage after 12,500 retrobulbar blocks. Anesth Analg 1993; 76: 1019-1022.

9. Sullivan KL, Brown GC, Forman AR, Sergott RC, Flanagan JC. Retrobulbar anaesthesia and retinal vascular obstruction. Ophthalmology 1983; 90: 373-377.

10. Cionni RJ, Osher RH. Retrobubar haemorrhage. Ophthalmology 1991; 98: 1153-1155.

11. Teichmann KD, Uthoff D. Retrobulbar (intraconal) anaesthesia with curved needle; technique and results. J Cataract Refract Surg 1994; 20: 54-60.

12. Davis DB, Mandel DM. Posterior peribulbar anaesthesia. An alternative to retrobulbar anaesthesia. J cataract Refract Surg 1986; 12: 182-184.

13. Rubin AP. Complications of local anaesthesia for ophthalmic surgery. Br J Anaesth 1995; 75: 93-96.

14. Loots JH, Koorts AS, Ventor JA. Peribulbar anaesthesia. A prospective statistical analysis of the efficacy and predictability of bupivaccine and lignocaine/bupivaccine mixture. J Cataract Refract Surg 1993; 19: 72-76.

15. Turbull CS. Editorial. Med Surg Rep 1884; 29: 628.

16. Swan KC. New drugs and techniques for ocular anaesthesia. Transactions of the American Academy of Ophthalmology and Otolaryngology 1956; 60: 368-375.

17. Fukasuku H, Marron JA. Sub-tenon pinpoint anaesthesia. $J$ Cataract Refract Surg 1994; $20: 673$.

18. Stewart MW, Lambrou FH Jr. Local anaesthesia for vitreoretinal surgery (letter). Arch Ophthalmol 1993; 111: 161.

19. Stevens JD. Curved, sub-tenon canula for local anaesthesia. Ophthalmic Surg 1993; 24: 121-122.

20. Redmond RM, Dalla NL. Extra capsular cataract extraction under local anaesthesia without retrobulbar injection. $\mathrm{Br} J$ Ophthalmol 1990; 74: 203-204.

21. Smith R. Cataract extraction without retrobulbar anaesthetic injection. Br J Ophthalmol 1990; 74: 205-207.

22. Peterson WC, Yanoff M. Subconjunctival anaesthesia: an alternative to retrobulbar and peribulbar techniques. Ophthalmic Surg 1991; 22: 199-201.

23. Khoo CY. Local anaesthesia without retrobulbar injection (letter) Br J Ophthalmol 1990; 74: 639. 
24. Jain VK, Lawrence M. Peribulbar versus retrobulbar anaesthesia. In: Jacobie FA, Adams AP, Volpe NJ, eds. Controversies in Ophthalmology: Current therapies and emerging technologies. International Ophthalmology Clinics. Boston: Little Brown and Company, 1994; 34-42.
25. Katsev DA, Drews RC, Rose BT. An anatomic study of retrobulbar needle path length. Ophthalmology 1989; 96: 12211224. 